\title{
Discours
}

Revue de linguistique, psycholinguistique et informatique. A journal of linguistics, psycholinguistics and computational linguistics

$7 \mid 2010$

Varia

\section{La co-construction textuelle avec de jeunes enfants : de la phrase au texte, et vice versa}

Frédéric Torterat

\section{OpenEdition}

Journals

Édition électronique

URL : http://journals.openedition.org/discours/8052

DOI : $10.4000 /$ discours. 8052

ISSN : 1963-1723

Éditeur :

Laboratoire LATTICE, Presses universitaires de Caen

\section{Référence électronique}

Frédéric Torterat, "La co-construction textuelle avec de jeunes enfants : de la phrase au texte, et vice versa », Discours [En ligne], 7 | 2010, mis en ligne le 20 décembre 2010, consulté le 19 avril 2019. URL http://journals.openedition.org/discours/8052 ; DOI : 10.4000/discours.8052

\section{(c) 1 (1) $\Theta$}

Discours est mis à disposition selon les termes de la licence Creative Commons Attribution - Pas d'Utilisation Commerciale - Pas de Modification 4.0 International. 

Revue de linguistique, psycholinguistique et informatique

\section{La co-construction textuelle avec de jeunes enfants : de la phrase au texte, et vice versa}

Frédéric Torterat

Université de Nice Sophia Antipolis/IUFM Célestin Freinet

EA 4080 (Paris-Sorbonne) / URE 03 (Nice)

frederic.torterat@unice.fr 



\title{
La co-construction textuelle avec de jeunes enfants : de la phrase au texte, et vice versa ${ }^{1}$
}

\author{
Frédéric Torterat \\ Université de Nice Sophia Antipolis / IUFM Célestin Freinet \\ EA 4080 (Paris-Sorbonne) / URE 03 (Nice)
}

\begin{abstract}
Cet article présente un type d'intervention pédagogique qui, menée avec de jeunes enfants, favorise les acquisitions simultanément dans les domaines de la structuration discursive et de l'organisation textuelle. Dans ces quelques pages, nous exposons les principes de la démarche en nous appuyant sur plusieurs extraits de corpus de terrain, ce qui nous conduit par la suite à montrer dans quelle mesure la construction phrastique contribue à l'organisation textuelle, et même l'anticipe à bien des égards, à travers en particulier la thématicité, la phoricité et la prédicativité. L'ensemble aboutit à déterminer ce qui, dans la co-construction textuelle qui s'établit, caractérise en particulier les moments spécifiques de la démarche et les interactions qu'elle permet d'engager entre le texte et la phrase.
\end{abstract}

Mots clés: phrase, texte, acquisition, co-construction, contexte pédagogique

This paper presents an educational intervention, with young children, which facilitates acquisition of both Discourse Structuring and Text Organization. In this article, we explain principles of this approach through several corpora, showing how Sentence Constructions contribute to Text Structuring, and even anticipate it. On the one hand, we examine thematic and anaphoric elements, but also predicates. On the other hand, we describe specific moments of this intervention to demonstrate to what extent it implies an interaction between Text and Sentences.

Keywords: sentence, text, acquisition, co-production, educational context

\section{Co-construire un texte avec de jeunes enfants: quelques principes}

\subsection{Un cadre acquisitionnel spécifique}

Les enfants sont aujourd'hui couramment confrontés, très tôt, à de multiples formes textuelles, dont ils s'approprient peu à peu la matérialité. Dans un contexte de socialisation, cette appropriation accompagne ce qu'on appelle l'acquisition du principe alphabétique, c'est-à-dire la compréhension du fait que les éléments graphiques renvoient à des matériaux phonologiques, et n'ont pas de signification par eux-mêmes. Par ailleurs, elle précède de quelques mois la capacité, chez les enfants, à produire individuellement des écritures dites «provisoires» (Jaffré, Bousquet et

1. Cette intervention a été présentée lors des premières Journées scientifiques $d u$ Crisco, organisées à Caen en décembre 2009. Nous remercions Denis Vigier, Didier Bottineau et Dominique Legallois pour leurs remarques et suggestions. 
Massonnet, 1999) ou "approchées» (Besse, 200I), lesquelles interviennent à partir de 46-50 mois mais ne concernent que des syllabes, des mots ou de sommaires constituants phrastiques.

Les productions textuelles à proprement parler, que nous envisagerons ici, en l'occurrence, comme des combinaisons organisées de phrases, prennent appui sur la reconnaissance et/ou l'emploi, par les enfants, d'assemblages récurrents de mots (Oller, 200o), qui sont en partie liés à des «patterns» lexico-syntaxiques (Veneziano, Sinclair et Berthoud, 1990; Tomasello, 2003), et prennent appui à l'oral sur des contours prosodiques diversement «unifiés» (Cresti et Moneglia (eds), 1993). Cela signifie, concrètement, que les jeunes enfants peuvent contribuer à la construction de diverses formes de textualité avant d'être en mesure de produire graphiquement des phrases brèves de leur propre initiative. Ces capacités s'accroissent avec l'apparition des premières diversifications syntaxiques, dont témoigne par exemple le recours à des constructions verbales ambitransitives ou à des opérateurs dont l'emploi se généralise au fil des années, jusqu’à conduire à la possibilité, qui apparaît quelquefois assez tôt chez certains enfants, de produire individuellement des textes brefs, comme celui-ci :

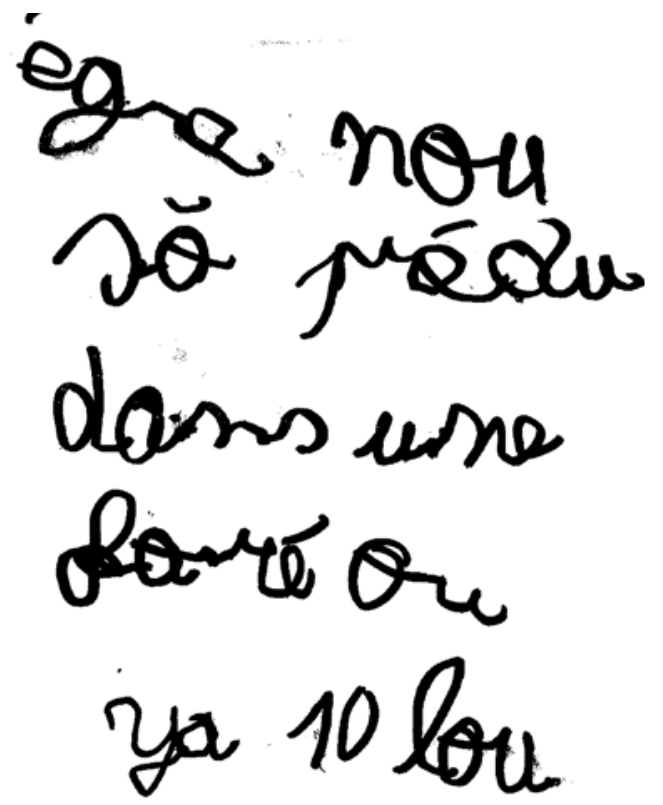

Cette production spontanée, qu'un entretien métagraphique (IO/I2/2009) nous a conduit à retranscrire en: Edgar/nous sommes perdus dans une forêt où il y a Io loups (Corpus J./2009-02), a été tracée sans aucune aide par un enfant de 7I mois ${ }^{2}$. Elle

2. Sur un ensemble de productions spontanées recueillies en contexte scolaire, lors d'exercices individuels de geste graphique, Nice, du 20/09/2009 au II/12/2009. 
comporte linéairement une apostrophe nominative, un pronom sujet, une forme verbale composée suivie d'un syntagme nominal prépositionnel qui contient luimême un «prédicat complexe» (Diessel, 2004; Bezinska, Chevrot et Novakova, 2008). Il y aurait plusieurs commentaires à formuler sur cette matérialisation intermédiaire, notamment d'un point de vue graphique (signes diacritiques, absence en partie légitime de «blanc graphique» dans l'amas locutionnel (il) y a et d'un $s$ pléonastique dans le pronom nous, etc.). Indiquons seulement qu'en plus de la présence d'un élément extraposé, de constructions prépositive et subordinative, de la présence d'opérateurs et d'une conformisation à certaines contraintes topologiques de précédence linéaire, nous assistons ici aux prémisses d'une organisation textuelle individuée.

Vers 36-6o mois, ces productions ne peuvent être que collectives, compte tenu du fait que, d'une part, le jeune enfant n'a pas la capacité d'accomplir la démarche individuellement, et du fait qu'il en est, d'autre part, aux tout premiers apprentissages du geste graphique et des correspondances grapho-phonologiques. Les productions textuelles, quand elles sont pratiquées sur cette période, sont donc proprement «dictées à l'adulte», pour reprendre l'expression de J. David, autrement dit co-construites avec l'accompagnant (qui écrit) et le collectif d'enfants impliqués dans la démarche. Sur le plan méthodologique, cela suppose que la prise en compte de la diversité des acquisitions porte autant sur des productions individuelles, que sur un «oral pluriel» (Bautier, 1997) déconstruit et reconstruit en interaction. Pour la recherche, les liens entre ces domaines de l'analyse sont facilités par le fait que la linguistique de l'acquisition présente des éléments communs avec la linguistique de corpus (à travers notamment le traitement et l'analyse des productions verbales), mais aussi la linguistique textuelle, avec laquelle elle entretient des liens effectifs. Qui plus est, quand il s'agit de traiter les co-verbalisations d'enfants en contexte pédagogique, plusieurs approches peuvent être mises en œuvre, comme l'approche intonosyntaxique, si la recherche concerne par exemple la question des liaisons phonologiques (Martel, 2009), ou statistique, si elle rassemble des données longitudinales en lien avec l'acquisition de la morphologie verbale (Bassano et al., 200I). Les analyses pratiquées, en outre, sont souvent multivariées (Diessel et Tomasello, 2005; Torterat, 20I0a), en ce qu'elles combinent plusieurs variables, qui sont autant de facteurs à envisager.

Remarquons que la co-construction textuelle n'est pas propre au cadre des apprentissages "premiers», ni à celui de l'apprentissage d'une manière générale (cf. le cas des «écritures collaboratives»). Ce qui nous occupe ici se résume toutefois en une pratique pédagogique qui consiste à faire construire par de jeunes locuteurs, à partir d'un canevas énumératif établi «en grand groupe», une textualité plus aboutie que les enfants «dictent à l'adulte» (Rébrard, 1987; Javerzat, 2004). Ces derniers le font en l'occurrence par «procuration graphique» (Torterat, 2009), suivant une méthodologie que nous présentons infra (en I.2.2). La dictée à l'adulte constitue donc la forme privilégiée de co-construction textuelle avec de jeunes enfants, d'autant qu'elle prend appui sur une mémoire commune dont des groupes restreints, de 3 à 6 sujets par exemple (dans certaines démarches pédagogiques), donnent des formulations distinctes suivant les capacités de chacun. 
Nous nous en tiendrons, pour les besoins de la démonstration, à des éléments de corpus discursifs collectés en contexte de formation (classes de maternelle), de manière à insister sur le fait que les interactions menées avec les enfants ne sont pas l'œuvre d'intervenant(e)s rompu(e)s à l'exercice, contrairement à ce qui pourrait être supposé à première vue. Les productions primaires ont été recueillies, généralement sans support filmique, auprès de groupes de ir à 27 enfants (ici de 46 à 65 mois), lors de séances pédagogiques de débat sur l'écrit à l'aide de matériels et de supports divers. Ces corpus rassemblent de 3000 à 6000 mots et sont instantanés (vs longitudinaux), et redressés, en ce qu'ils incluent un minimum de disfluences propres à l'oral (répétitions intempestives, hésitations en particulier), de même qu'ils ne reprennent pas les interventions inaudibles (cf. Torterat, 2orob). Nous insisterons donc sur ce qui caractérise la pratique elle-même ainsi que les supports employés, en exemplifiant dans quelle mesure la phrase conditionne la production textuelle, tout en l'anticipant, dans une interaction qui s'appuie, en particulier, sur des moments spécifiques sur lesquels nous apporterons quelques éléments de conclusion pour la recherche.

\subsection{Principales caractéristiques de la démarche}

\subsubsection{Une prise en compte des représentations individuelles}

L'une des principales caractéristiques de la co-construction textuelle est qu'elle conduit à confronter les multiples représentations que les enfants se font de l'écrit. Celles-ci portent, d'une part, sur les éléments graphiques (comme les cédilles, les combinaisons trigrammatiques, mais aussi les «blancs»: Froment et Leber-Marin, 2003) et sur la ponctuation (laquelle est plus ou moins démarcative: Chauveau et Rogovas-Chauveau, I990; Dahlet, 2003). D'autre part, les représentations concernent certaines données prosodiques, comme les groupes intonatifs (lesquels rassemblent de 2 à 4 mots ou, pour être plus exact, de 2 à 7 syllabes), d'autant que ces derniers coïncident variablement, dans les productions verbales, avec «l'organisation de l'information discursive» (Mertens, 2006: 65). En outre, les enfants se familiarisent peu à peu avec des opérations (comme la détermination, la quantification) et des constructions (factitives, coordinatives, prépositives, etc.) qui, à l'écrit, se matérialisent de manière spécifique et ne correspondent qu'en partie, dans bien des cas, à ce qui est proprement produit à l'oral. Le fait par exemple, que la négation soit bitensive à l'écrit, suppose qu'elle y soit plus ou moins pléonastique. De même, le fait que l'extraposition d'un élément discursif ne soit pas envisageable à certains moments de la phrase montre que tous les éléments ne sont pas forcément déplaçables. Rappelons en revanche, à titre anecdotique, que la plupart des intervenant(e)s de terrain, en particulier les professeurs des écoles, font spontanément porter la réflexion sur les compléments du verbe, à travers les transitivités directe et indirecte, mais aussi la présence de clitiques ou la distinction entre compléments conjoints et adjoints (comme le sont, pour les adjoints, de nombreux circonstants) ${ }^{3}$. Mais cela s'effectuant sans exclusive, d'autres domaines de questionnement sont abordés.

3. Les professeurs des écoles n'en font pas, bien entendu, des séances spécifiques en maternelle (ce qui serait pour le moins incongru!), mais s'en saisissent à des fins d'initiation, comme le recommandent 
L'autre principale caractéristique de la co-construction textuelle est qu'elle oscille entre co-locution et reformulation. La co-locution, en particulier, revient, du côté de l'intervenant en charge de l'écrit comme de celui des enfants, soit à favoriser l'emploi (à plusieurs) de tel ou tel élément discursif, soit à l'extraire de ce qui vient d'être dit, aboutissant ainsi à une compilation d'éléments phrastiques qu'il s'agit peu à peu d'organiser dans une forme de textualité, comme l'exemplifie l'extrait suivant ${ }^{4}$ :

\section{$\mathrm{M}$ : il était/une fois}

Léonie: le petit chaperon rouge

Tessa: il se promena

$\mathrm{M}:$ il se promena/et rencontra?

El. : le loup/le loup

M: et rencontra/le loup// et qu'est-ce qu'il lui dit?

Tessa: tu vas par ce chemin/et moi je vais passer par ce chemin// le petit chaperon rouge se promena// très longtemps

Kimberly: et le loup se promena/et il rentra à la maison

Tessa: tire la chevillette

$M$ : et il rentra

Kimberly: la grand-mère

Tessa: c'était la grand-mère

$\mathrm{M}$ : et qu'est-ce qui se passe?

Léonie: il le mange

(DONog)

Cet extrait montre que les enfants s'approprient variablement plusieurs mécanismes d'organisation textuelle, comme l'emploi de marqueurs spécifiques tels que $e t$ (qui indique à la fois la coordination grammaticale et la concaténation discursive), le recours à des formes verbales tensées (qui inscrivent les événements dans une trame linéaire), et le réemploi, avec une formulation plus ou moins aboutie, de quelquesunes des expressions énumérées dans le canevas commun (cf. infra). Placé(e) à côté du support élaboré en grand groupe, l'intervenant(e) le déconstruit et le reconstruit à l'aide des enfants, amenant ainsi les locuteurs à produire le début d'un texte provisoire (ici il était une fois, il se promena et rencontra le loup, et il rentra), qu'il s'agira d'amender et de compléter au fur et à mesure des séances qui lui sont consacrées.

Pour ce qui concerne la reformulation, que nous exemplifierons dans les rubriques suivantes, celle-ci permet notamment de rapporter l'énoncé dicté à l'énoncé source, lequel est non seulement repris, mais modifié à la faveur de corrections suscitées par des exigences qui interviennent au fur et à mesure de la démarche de textualisation.

d'ailleurs clairement les Programmes de l'École primaire (2007, 2008).

4. L'abréviation « $\mathrm{M}$ » renvoie ici à l'adulte. Par commodité, nous indiquerons en gras dans tous les extraits, comme dans celui-ci, ce qui est proprement conservé par écrit, et donc porté sur le support (papier, ardoise, tableau, etc.). Les abréviations instruites à la suite de chaque extrait identifient la source du corpus. Ces ressources ont été constituées à l'occasion de suivis de stages «filés» et "groupés» de formation des professeurs des écoles, dans l'académie de Nice (2002-2009), et sont archivées à l'IUFM Célestin Freinet. 
La reformulation précise d'autre part les contours des implications interpersonnelles qui apparaissent lors du dialogue, rendant à l'exercice la dimension pédagogique qui lui revient. Dans l'extrait ci-dessus, les modifications ont ainsi porté sur l'événementialisation des actions narratives (à l'aide de circonstants par exemple), mais aussi sur la désambiguïsation du pronom il dans le SV il rentra, où l'anaphorique pose problème en termes de compréhension textuelle.

Confrontés à toutes ces informations simultanément, les enfants, à partir de leurs représentations, deviennent à la fois les contributeurs d'un texte en construction, et les remarqueurs de ce que nous appellerons assez simplement des faits récurrents, lesquels touchent autant à la phrase qu'au texte lui-même.

\subsubsection{La phrase anticipatrice du texte}

La production commune des «canevas», à partir desquels les groupes de participants vont co-construire les textes, s'inscrit dans un moment pédagogique particulier, dans la mesure où ces énumérations d'expressions et de phrases permettent d'anticiper en partie ce que seront les contenus et les formes proprement «dictés» par les enfants. Nous en relevons une trace significative à partir de l'extrait reporté ci-dessus, en I.2.I, quand Tessa formule ceci:

tu vas par ce chemin/et moi je vais passer par ce chemin// le petit chaperon rouge se promena// très longtemps

Repris indirectement du canevas produit par le groupe classe, cet énoncé répond à la question de «M» qui précède («et qu'est-ce qu'il lui dit?»). La réponse, en quelques mots, passe du cadre interlocutif des propos rapportés au cadre délocutif de la narrativisation à la troisième personne, que Tessa circonstancie à l'aide d'un adverbe temporel combiné à un intensif. La jeune locutrice répond à la commande qui consiste à prendre appui sur les éléments préconstruits, tout en les réaménageant (et donc en les reformulant). À cette occasion, elle produit des phrases qui lui sont personnelles, mais qui s'inscrivent dans une organisation théma-rhématique en partie caractérisée par des fragments textuels mémorisés. Ici, l'enfant a repris les expressions «(le) chemin», «le petit chaperon rouge» et «se promene(r)» en les répartissant entre les propos rapportés et la narration qui les encadre: de ce fait, elle a démontré sa capacité à intégrer pour le moins, dans sa production discursive, les paramètres de la thématicité, de l'encadrement diégétique et de l'interlocution.

D’une manière générale, les canevas rassemblent de 15 à 70 mots, eux-mêmes regroupés la plupart du temps dans quelques inventaires ou quelques phrases sommaires. En voici un exemple, repris de MARo7:

$$
\begin{aligned}
& \text { NOTRE HISTOIRE } \\
& \text { - graine } \\
& \text { - petite } \\
& \text { - marron } \\
& \text { - enterrée mais pas germée }
\end{aligned}
$$


- toutes les autres graines ont germé, et elle s'ennuie

la graine s'en va sur la plage

- elle voit une île au loin

elle rencontre un dauphin qui l'amène sur l'île

Cette énumération, où la matérialité phrastique est irrégulièrement représentée, rassemble une intitulation intermédiaire, suivie d'un paragraphe qui comporte un inventaire descriptif et une contradiction diégétique (non narrativisée). Le deuxième sous-ensemble paragraphique contient un répertoire événementiel semi-narrativisé qui permet de poser l'exigence de constructions parataxiques et hypotaxiques, lesquelles sont combinées ici à des formes verbales composées, mais aussi pronominales et participiales. Les entretiens avec les intervenants et les enregistrements effectués témoignent du fait que, lors de la production de tels canevas, les matériaux pris en compte conduisent à sensibiliser les jeunes locuteurs à l'existence - en plus de la diversité des constructions phrastiques possibles - de faits d'ordre phonologique, comme c'est le cas de l'amuïssement («germé(e)») ou plus particulièrement de l'élision («NOTR(E) HISTOIR(E)», «îl(e) au loin»), à savoir que la liaison est elle aussi couramment sollicitée.

En complément, voici un autre canevas:

un dragon qui crache du feu.

un vampire qui se balade et qui rencontre une sorcière.

la sorcière qui le transforme en crapaud

et le vampire qui lui suce le sang. Un roi qui voulait tuer tout le monde.

(BLA/GERo7)

Cette série à la fois descriptive et narrative, qui tient du répertoire, apporte de multiples indications sur les actants de la narration à produire et sur la trame événementielle esquissée par les participants. Elle permet aussi de familiariser les jeunes enfants avec l'emploi d'une construction hypotaxique, du type [SV [pronom subordonnant/V/(OBJET)]]. Outre l'organisation diégétique que ce canevas conduit à envisager, les enfants sont sensibilisés à la dimension microsyntaxique des énoncés à travers par exemple la pronominalisation, l'ellipse et l'hypotaxe, mais aussi à leur dimension macrosyntaxique, à travers notamment la présence d'une zone préverbale à laquelle correspondent des éléments focaux, ici tous thématiques et tous repris par une proforme sujet.

La préconstruction d'une forme de textualité sommaire à l'aide de quelques phrases, elles-mêmes intermédiaires, influence donc variablement la construction de celles qui, produites oralement par les enfants, seront proprement «dictées» à l'intervenant(e). Ces questions concernent à la fois la parataxe et l'hypotaxe, mais aussi la transitivité verbale, ou encore la prise en compte des modifications diathétiques (il n'y a rien d'exceptionnel à ce que les enfants de 50-60 mois emploient, spontanément, une construction verbale passivée). Dans une approche à la fois linéaire et récursive, la production textuelle transite alors par plusieurs «révisions» 
(Schubauer-Léoni, 1997), amenant de ce fait la phrase à devenir un objet de détermination textuelle, tout en anticipant le texte, dans un va-et-vient constant entre la formulation et sa correction, mais aussi, du point de vue des enfants, entre l'informulé, le formulé et le reformulé.

Les constructions phrastiques émergent de ce fait, en grande partie, des modes organisationnels informés par le canevas textuel, à partir duquel les locuteurs, grâce à une démarche pédagogique spécifique à ce type d'action, rétablissent une forme d'aller-retour pour le moins productif, comme nous allons le voir ci-après.

\section{Phrase et textualité en interaction}

\subsection{Thématicité, phoricité et prédicativité}

Dans une approche constructiviste, et à bien des égards socio-constructiviste, où nous nous plaçons ici, il convient de rappeler que le passage collectif à l'écrit, pour des enfants qui ne sont donc pas encore en mesure de le concrétiser par eux-mêmes, aboutit à des matérialités phrastiques et textuelles qui leur sont plus ou moins accessibles individuellement. Les intervenant(e)s passent donc, outre les canevas que nous avons évoqués, par l'identification de certains faits grammaticaux qu'ils ne nomment pas, bien entendu, mais dont ils relèvent l'existence à l'aide d'exemples et de manipulations, ainsi que par le recours à des conduites verbales familières chez les enfants, comme le sont la narrativisation, la description et la citation. À ce titre, les incitations se multiplient aussitôt que les locuteurs paraissent en difficulté:

$\mathrm{M}$ : je vous relis la phrase et vous m'arrêtez/quand vous pensez que c'est le bon endroit/pour le le mettre// la petite graine appela le dauphin// il se mit près de petite graine/et la petite graine monta sur l'aileron

$\mathrm{E}: \ldots$

$\mathrm{M}$ : on peut le rajouter à la fin de cette phrase-là// je relis la phrase et vous la complétez

Victoire: pour aller voir d'autres petites graines/sur l'île

(MARo7)

Cette intervention, qui s'appuie sur l'un des canevas précédemment cités, littéralement «met en phrases» des fragments textuels énumérés qu'il s'agit de «compléter»: les principaux éléments thématiques de la chaîne discursive ayant été identifiés, la thématicité s'assortit, aussitôt que la chaîne se linéarise au fur et à mesure des participations, d'un processus de phoricité que l'intervenant(e) inscrit généralement dans une gradation. Ainsi l'élément rhématique le daupbin (dans l'énoncé dicté) devient-il l'élément thématique du syntagme verbal subséquent à travers les pronoms $i l$ et $s e$, une pronominalisation que l'intervenant(e) n'ose pas appliquer à l'élément la petite graine, qui est juste répété pour ne pas accumuler les éventuelles difficultés. Cela lui permet d'insister sur la complémentation verbale, à laquelle Victoire fait 
correspondre deux circonstants, alors même qu'elle segmente ses propres énoncés par des frontières prosodiques plus ou moins marquées (cf. Banel et Bacri, 1997, qui parlent d'«indices de segmentation»).

De telles sollicitations sont particulièrement profitables pour les jeunes enfants (ci-dessous de $5 \mathrm{r}$ à 62 mois), comme en témoigne l'extrait suivant:

Chloé: on pourrait l'emmener au gymnase

M: vous vous rappelez ce qu'il faisait/dans le jardin d'enfants?// qu'est-ce qu'il peut faire au gymnase? [...]

Chloé: il va dire oui/parce qu'on va s'amuser

$\mathrm{M}$ : qu'est-ce qu'on va lui dire après?

Chloé: moi/je lui dis// tu veux t'amuser avec nous?

$\mathrm{M}$ : alors/comment on l'emmène?// on lui donne la main/on lui explique par où il doit passer?

Lina: on lui donne la main/et on se met derrière// pour faire le bus

$\mathrm{M}$ : alors tous les enfants lui donnent la main?

Michel: on peut le laisser trainer une corde/et il nous suit

(FOR/KLEO2)

Ce passage illustre de quelle manière l'intervenant(e) suggère l'existence de plusieurs formes d'événementialisation, auxquelles souscrivent volontiers les jeunes locuteurs. Chloé et Lina accompagnent ainsi les sollicitations de l'adulte en montrant qu'elles ont saisi qu'un moment textuel consistera tantôt dans une narrativisation (répliques I-2 [Chloé-M] et 6-9 [M-Lina-M-Michel]), tantôt dans le recours aux propos rapportés (que Chloé s'approprie d'ailleurs personnellement). La présence d'opérateurs intradiégétiques, tels que et du côté des enfants, et alors du côté de l'intervenant(e), indique une concaténation d'événements qui passe, dans le domaine de la phrase, par des constructions principalement parataxiques.

La prise en compte de la thématicité s'établit à partir d'éléments plus ou moins focaux, mais donc aussi d'opérateurs que nous appellerons volontiers des thématisants, comme et (lui il_), voici_ou c'est_(qui). Sur le plan de la prosodie phrastique, cela revient généralement à rassembler des indications de la zone préverbale (que les enfants intonent d'ailleurs avec plus ou moins d'intensité), en lui donnant plus de consistance par la suite. Qui plus est, la répartition qui s'effectue entre déterminants et noms, verbes et sujets, compléments conjoints et compléments adjoints implique directement des questions traitant de la prédicativité des éléments relevés, notamment sur la distinction qui s'impose d'elle-même entre les éléments noyaux et ceux qui s'organisent autour d'eux, comme les syntagmes infinitivaux ou gérondivaux, les appositions ou les circonstants. Il en est de même concernant la phoricité, qui passe par diverses opérations de substitution (par pronominalisation le plus souvent), mais qui conduit aussi les intervenant(e)s à initier les jeunes locuteurs aux questions de rattachement et d'appariement, au moment par exemple où il s'agit d'éliminer dans les phrases dictées, lors des révisions textuelles, les répétitions assimilables à des 
redondances et bien sûr les constructions tautologiques, que les enfants corrigent assez spontanément. Ces derniers s'approprient, au fil d'une pratique quelquefois hebdomadaire dans certaines classes de maternelle, ce rapport dialectique qui s'opère entre la construction phrastique et l'organisation textuelle:

$\mathrm{M}$ : alors Chloé/pourquoi tu veux lui donner des bonbons?

Chloé: parce qu'il s'est fait mal

$\mathrm{M}$ : on va essayer de faire des phrases/tous ensemble

Chloé: on va lui donner des bonbons

$\mathrm{M}$ : est-ce qu'il vous a appelé?

E: oui [ non

M: qu'est-ce qu'il vous a dit?

Vincent: aidez-moi/aidez-moi à me relever

$\mathrm{M}$ : qui crie ça?

Vincent: l'ours

M: aidez-moi// aidez-moi à me relever/crie l'ours // qui va l'aider à se relever? / estce qu'un seul peut le faire?

EI, 2: non

M: tous les enfants//

Raphaël: et leurs parents

$\mathrm{M}$ : tous les enfants et leurs parents// aidez-moi à finir la phrase

Jordan: vont l'aider à se relever

$\mathrm{M}$ : tous les enfants et leurs parents vont l'aider/à se relever// est-ce que vous avez encore peur quand vous allez le relever?

E: oui [ non

$\mathrm{M}:$ que dit l'ours?

EI, 2, 3: merci les enfants

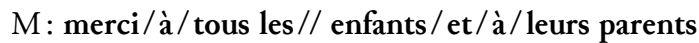

Margaux : s'il pleurait/on lui donne des bonbons

$\mathrm{M}$ : oui/alors s'il pleure/comment on dit?// pourquoi on lui donne des bonbons?

Jimmy: pour le remercier

$\mathrm{E} 2$ : pour le pardonner

(FOR/KLEo2)

L'exigence de la textualisation ayant été posée précédemment, celle qui consiste à «faire des phrases» à partir de quelques éléments thématiques incite ici l'intervenant(e) à accumuler les éléments verbaux (donner, appeler, dire, crier, etc.), afin que les locuteurs s'en saisissent comme les noyaux organisateurs des énoncés dictés. Les éléments thématiques coïncidant généralement avec les sujets grammaticaux, de manière non pas à simplifier les phrases, mais à différer leur diversification à d'autres moments pédagogiques (comme les révisions), c'est sur la complémentation verbale que porte principalement le dialogue entre les participants. D’un côté, les sujets grammaticaux sont presque tous informés par un répertoire préétabli, et de l'autre, les noyaux prédicatifs sont en partie suscités par l'intervenant(e), qui laisse par là même aux jeunes locuteurs la possibilité de compléter les verbes soit par des 
éléments co-prédicatifs (tels que des formes adjectivales du verbe), soit, le plus souvent, par des compléments conjoints ou adjoints. Une telle aide, qu'on appelle à juste titre l'«étayage» en pédagogie comme en acquisition (cf. Hudelot, 1999), a pour objectif de permettre aux enfants, comme nous l'avons précédemment suggéré, de s'approprier deux démarches simultanément: celle de la construction phrastique et celle de l'organisation textuelle. En ce qui concerne cette dernière et pour ce qui relève de l'extrait ci-dessus, les jeunes locuteurs sont invités à rapporter les propos des personnages, sous forme de discours direct (d'abord «libre» de toute ponctuation sur le support), que les enfants reprennent en s'appuyant pour certains sur des prédicats complexes (Vincent: «aidez-moi à me relever»), sur des objets grammaticaux (Margaux: «on lui donne des bonbons») ou sur des circonstants (Jimmy: «pour le remercier»).

De telles interactions, outre une représentation en pratique du geste graphique et une consolidation progressive du principe alphabétique et des correspondances grapho-phonologiques, conduisent les enfants à saisir que les éléments thématiques peuvent être repris dès lors qu'une chaîne de phoricité s'établit, mais aussi que les verbes tensés, qui sont généralement les noyaux prédicatifs de la chaîne, peuvent être complétés par des prédicats plus ou moins diversifiés. Le texte lui-même n'apparaît pas comme un simple regroupement de constituants phrastiques, mais dans le rapport dialectique qu'il entretient avec la phrase, laquelle a ses propres exigences et apporte une contribution variable à ce qui forme la cohésion textuelle.

\subsection{La démarche de textualisation: des moments spécifiques}

En résumé, nous pouvons distinguer plusieurs moments spécifiques au processus à l'œuvre. D'une part, la co-construction textuelle, au moment de la préconstruction des formes de textualité, consiste en un regroupement de nominations et de prédications intermédiaires, une événementialisation sommaire, et la reconnaissance d'une topologie phrastique de types sujet/verbe et verbe/compléments. D'autre part, au moment, précisément, des "premières phrases», les co-productions portent sur la construction de prédicats complexes (noms déterminés et caractérisés, verbes tensés suivis ou précédés de compléments conjoints/adjoints), sur leur diversification (coordination et subordination, citations directe et indirecte), et sur une topologie phrastique plus variée, avec des clitisations et des extrapositions de quelques éléments focaux. Enfin, la co-construction textuelle, au moment des phrases révisées, tient compte de certains contours intonatifs et de certaines frontières prosodiques à l'oral pour ponctuer les énoncés, et en appelle à une événementialisation plus aboutie, mais aussi à la sélection, dans un «ensemble de formulations» (Siblot, Détrie et Verine, 200I), des éléments discursifs les plus significatifs pour une meilleure compréhension du texte.

Pour insister sur le fait que ces processus sont en lien les uns avec les autres, tout comme c'est le cas de la phrase et du texte (et vice versa), nous prendrons deux exemples de dialogues que suscite une réflexion conjointe entre l'intervenant(e) et 
les enfants sur les versions à donner de phrases intégratrices du texte. La première illustration relate une réaction d'enfant sur une extraction pléonastique, et la deuxième sur les modifications syntaxiques impliquées par la transition d'une suite énumérative à une chaîne narrativisée.

\section{Voici l'extrait I :}

Nicolas: elle aime bien se regarder dans l'eau du lac

Marius: Coquette

Ciana: quand elle se regarde dans l'eau

$\mathrm{M}$ : alors/qu'est-ce que j'écris?

Louis: Coquette/elle aime bien se regarder dans l'eau du lac

$\mathrm{M}$ : bon j'écris/Coquette, elle aime

Marius: non c'est pas ça

$\mathrm{M}$ : c'est quoi alors?

Marius: Coquette aime bien//et ensuite se regarder

Ciana: dans l'eau du lac

M (ayant effacé le tableau): vas-y/Nicolas//Coquette COQUETTE

Nicolas: aime bien se regarder

M: aime bien se regarder/AIME BIEN SE REGARDER

Marius : et ensuite tu mets dans l'eau du lac/voilà

(GERo3)

Alors que les jeunes locuteurs semblent s'être approprié certaines exigences propres à la grammaticalité des phrases, et donc aux contraintes de bonne formation (liens déterminants/noms, verbes/compléments), et parmi elles celles qui ont une implication directe dans l'organisation textuelle ('appariement en particulier), certains d'entre eux corrigent mutuellement leurs énoncés. En effet Marius, alors que l'intervenant(e) semble disposé(e) à écrire sur le support l'énoncé dicté par Louis («Coquette, elle aime bien se regarder dans l'eau du lac»), corrige une extraction qui lui parait erronée («non, c'est pas ça»). Cette sensibilité à l'exercice (et à la phrase!) est d'autant plus remarquable chez Marius qu'il fait pour ainsi dire un emploi métalinguistique des opérateurs et ensuite, à deux reprises, non pas sur le plan phrastique, mais sur celui de l'organisation textuelle (et même graphique). Sur le plan pédagogique, de telles conduites interlocutives permettent de faciliter les démarches collaboratives, d'individualiser la prise en compte des difficultés, mais aussi d'organiser les groupes selon les capacités des uns et des autres. Ce que l'«initiation» pose à ce moment-là, s'appuie autant sur ce qu'osent les enfants, que sur ce qu'ils en présument.

Une autre question est liée au fait que la narrativisation reste une opération plurielle et que, dans les interventions des enfants, elle s'assortit de commentaires plus ou moins contextualisés. Les variations interindividuelles apparaissent à ce titre à travers les capacités des jeunes contributeurs à souscrire à la démarche de textualisation elle-même, mais aussi à travers leur appropriation de ce que Chiss et Meleuc (200I) nomment la «constructibilité» phrastique, comme le démontre l'extrait qui suit: 
M: quel jour ça se passe?// vous vous souvenez?

Inès: oui/c'est jeudi/parce qu'il a déjà vu les mangoustes rayées// et les petites// et puis il s'est cassé l'œuf sur la tête

$\mathrm{M}$ : oui/on a déjà vu trois endroits qu'il a visités

Aurélia: moi je sais/parce que tu l'as lu tout à l'heure

$\mathrm{M}$ : oui Aurélia/tu as raison// alors je marque// jeudi // où se trouve Sunny à votre avis?/Fanny?

Fanny: il est sous la pluie/il pleut

$\mathrm{M}$ : d'accord/il pleut// qu'est-ce qu'on peut écrire alors?

Théa: on peut marquer/comme la dernière fois// le jeudi//

Fanny: puis/il pleut

M: d'accord/j'écris/le// jeudi// il// pleut// alors dans quel endroit est Sunny?/ qu'est-ce qu'il a autour de lui ?/Antonio?

Antonio: moi je vois les arbres

Lili: c'est une forêt// y a beaucoup d'arbres

$\mathrm{M}$ : oui Lili// et que fait-il?

Flore: il tient sa valise/sur la tête

Yann: il pleut/il se protège de la pluie// la pluie/ça mouille

$\mathrm{M}$ : c'est vrai Yann// alors on va l'écrire// tu peux me répéter ça/Flore?

Flore: il tient sa valise/sur sa tête

$\mathrm{M}$ : on peut le dire autrement?

Fanny: il s'abrite

M: oui/alors on va dire/Sunny//

Flore: s'abrite avec sa valise

M: très bien/j'écris// alors// et/Sunny// s'abrite// avec// sa/valise// je relis/le jeudi/il pleut et Sunny s'abrite avec sa valise

Julien: moi j'aime pas la pluie

M: ah/Julien// tu me dis que tu n'aimes pas la pluie// mais tu crois que Sunny aime la pluie?

Julien: non// mais y a un parapluie// là

$\mathrm{M}$ : oui/mais ce n'est pas Sunny qui le tient// qui tient le parapluie?

EI, 2, 3: c'est les mangoustes

(LEFo9)

Le début de ce dialogue indique que la nomination, qui prend donc appui sur un répertoire commun, se combine peu à peu avec la détermination, mais aussi que les enfants narrativisent les événements au gré de remarques et de commentaires qui leur permettent de s'approprier la linéarité du récit, et donc de se représenter simultanément la trame diégétique. L’intervenant(e) incite les locuteurs à s'extraire d'une formulation trop énumérative pour aboutir à une production textuelle plus condensée, pour ainsi dire plus proche de l'écrit.

Constamment, l'objectif du geste graphique («alors je marque», «j'écris») s'allie à celui de la co-locution («qu'est-ce qu'on peut écrire alors?», «on peut marquer») et à celui de la reformulation («tu peux me répéter ça?», "on peut 
le dire autrement?»), formant ainsi une combinaison de mécanismes qui, non seulement favorisent les acquisitions, mais préviennent en un sens d'éventuelles régressions. Le caractère à la fois provisoire et sommaire de la production commune (le jeudi, il pleut, et Sunny s'abrite avec sa valise) peut éventuellement poser question, mais n'oublions pas que chacun des groupes ou que le groupe «classe» ayant construit ses propres formulations, ce type d'exercice multiplie, quand il est pratiqué régulièrement, les familiarisations avec l'écrit, et donc les acquisitions envisageables dans ce domaine. En outre, l'approche intégralement interlocutive permet non seulement de prendre en compte les variations interindividuelles parmi les locuteurs, mais aussi leur implication dans une démarche qui appartient autant au groupe qu'aux personnalités de chacun.

\section{En conclusion}

Même si elle est pratiquée depuis plusieurs années et qu'elle mérite de se combiner avec d'autres types d'actions, la co-construction textuelle demeure un exercice pédagogique particulièrement productif, en termes de facilitation et d'acquisition ${ }^{5}$. Tout en favorisant une première appropriation des spécificités de l'écrit, elle s'appuie sur les représentations qu'en ont les jeunes enfants, tant au niveau de la phrase qu'à celui du texte. Elle les incite dans le même temps à comprendre que pour une même opération (prédication, détermination ou modification: Desclés, 2009), plusieurs constructions phrastiques sont envisageables, et qu'il en est de même pour ce qui garantit la cohésion discursive des éléments prédiqués (cf. Sanders, Spooren et Noordman, 1992). La préconstruction textuelle, telle qu'elle s'établit dans ce cadre, littéralement «rend aux phrases» toutes leurs dimensions, à savoir une «constructibilité», une matérialité à la fois topologique, prosodique et graphique, mais aussi une grande diversité.

Dans bien des cas, les regroupements d'expressions verbales coïncident avec des «patterns» (cf. I.I) qui influencent en même temps les constructions phrastiques et l'organisation textuelle, mais à des titres divers, ce qui marginalise l'entrée pédagogique d'une "grammaire textuelle», avec ce qu'elle comporte d'exceptions contre-productives (Torterat, 20IOc). Certains des éléments pris en compte confirment d'ailleurs que les moments textuels envisagés s'inscrivent dans un paradigme interactionnel où plusieurs «actions spécifiques», pour reprendre l'expression de Fox (2007), émergent d'un réseau d'intercompréhensions entre les participants, mais aussi d'une certaine approche de la matérialité textuelle et de ce que la phrase, à ce titre, procure au texte. Il conviendrait sans doute, d'autre part, de confronter les éléments «dictés» à l'existence possible, en termes d'organisation phono-prosodique du discours, d'«unités périodiques» (Roulet, 1999: 8I) coïncidant variablement avec les groupes intonatifs sur lesquels s'appuie la co-construction textuelle. Il en est

5. Concernant les déductions qu'il est possible de formuler sur ces profils d'action plus généralement sur le plan développemental, voir notamment Negro et Chanquoy (2005) ainsi que Veneziano et Hudelot (2007). 
de même pour les contributions respectives de la thématicité et de la phoricité en particulier, lesquelles représentent des variables peut-être plus significatives que ne le sont les caractéristiques catégorielles (noms et verbes notamment) en acquisition. Mais ce sont là quelques pistes parmi d'autres, lesquelles rejoignent toutes ce que la textualisation rassemble de «faits marquants» et de «moments critiques».

\section{Éléments de bibliographie:}

BANEL, M.H. et BACRI, N. 1997. Reconnaissance de la parole et indices de segmentation métriques et phonotactiques. L'Année Psychologique 97: 77-II2.

Bassano, D. et al. 20or. L'acquisition de la morphologie verbale à travers les langues. Enfance 53 (I) : 8I-99.

BAUTIER, E. 1997. Les pratiques socio-langagières dans la classe de français. Quels enjeux? Quelles démarches? Repères 15 : II-25.

BEsSE, J.M. 20or. L'accès au principe phonographique: ce que montrent les écritures approchées. In G. Chauveau (ed.), Comprendre l'enfant apprenti lecteur. Paris: Retz: $130-158$.

Bezinska, Y., Chevrot, J.P. et Novakova, I. 2008. Le prédicat complexe faire + Vinf dans le langage enfantin entre 4 et 6 ans. In J. Durand, B. Habert et B. LaKs (eds), Actes du premier Congrès mondial de linguistique française. Paris: Institut de linguistique française: 1707-1720.

Chauveau, G. et Rogovas-Chauveau, E. 1990. Les processus interactifs dans le savoir-lire de base. Revue Française de Pédagogie 9o: 23-30.

Chiss, J.L. et Meleuc, S. (eds) 200I. Le Français aujourd'hui: Et la grammaire de phrase? 135. Paris: Larousse.

Cresti, E. et Moneglia, M. (eds) 1993. Ricerche sull'acquisizione dell'italiano. Rome: Bulzoni.

DAHLET, V. 2003. Ponctuation et énonciation. Matoury: Ibis Rouge Éditions.

Desclés, J.P. 2009. Prédication en logique et en linguistique, une approche cognitive et formelle. In A.H. ІвRAнiм (ed.), Prédicats, prédication et structures prédicatives. Paris: Cellule de recherche en linguistique: 82-III.

Diessel, H. 2004. The Acquisition of Complex Sentences. Cambridge Studies in Linguistics I05. Cambridge - New York: Cambridge University Press.

Diessel, H. et Tomasello, M. 2005. Particle Placement in Early Child Language: a Multifactorial Analysis. Corpus Linguistics and Linguistic Theory I (I): 89-III.

Fox, B. 2007. Principles Shaping Grammatical Practices: An Exploration. Discourse Studies 9: 299-318.

Froment, M. et Leber-Marin, J. 2003. Analyser et favoriser la parole des petits. Issy-lesMoulineaux: ESF Éditions.

HudeLot, C. 1999. Étayage langagier de l'enseignant dans le dialogue maître-élève. In M. Gilly, J.-P. Roux et A. Trognon (eds), Apprendre dans l'interaction. Nancy: Presses universitaires de Nancy: 219-240.

Jaffré, J.-P., Bousquet, S. et MAssonnet, J. 1999. Retour sur les orthographes inventées. Les Dossiers des sciences de l'éducation I: 39-52. 
JAVERZAT, M.C. 2004. La dictée à l'adulte comme genre de l'activité scolaire d'apprentissage du langage écrit. Mélanges CRAPEL 29:87-ı09.

Martel, K. 2009. Unités prosodiques et premières combinaisons de mots chez des enfants âgés de 30 mois. Travaux linguistiques $d u$ Cerlico 22: II-2I.

Mertens, P. 2006. A Predictive Approach to the Analysis of Intonation in Discourse in French. In Y. Kawaguchi, I. Fonagy et T. Moriguchi (eds), Prosody and Syntax. Amsterdam - Philadelphia: John Benjamins: 64-гог.

Negro, I. et Chanquoy, L. 2005. The effect of psycholinguistic research on the teaching of writing. LI-Educational Studies in Language and Literature. Writing: Contributions from Applied and Fundamental Research to the Teaching of Writing 5 (2): IO5-III.

Oller, D.K. 200o. The Emergence of the Speech Capacity. Mahwah: Lawrence Erlbaum Associates.

RÉBRARD, T. 1987. Dictée à l'adulte et énonciation écrite au cours préparatoire, étude linguistique. In L. Lentin (dir.), Recherches sur l'acquisition du langage I. Paris: Université de la Sorbonne Nouvelle: I69-ı80.

Roulet, E. 1999. Une approche modulaire de la complexité de l'organisation du discours. In J.M. AdAM et H. Nølke (eds), Approches modulaires: de la langue au discours. Lausanne : Delachaux \& Niestlé: : $87-257$.

Sanders, T., Spooren, W. et Noordman, L. 1992. Toward a Taxonomy of Coherence Relations. Discourse Processes I5 (I): I-35.

SCHUBAUER-LÉONI, M.L. 1997. Interactions didactiques et interactions sociales: quels phénomènes, quelles constructions conceptuelles? Skoblê, cabiers de la recherche et $d u$ développement 7: 103-134.

Siblot, P., Détrie, C. et Verine, B. 200I. Termes et concepts pour l'analyse de discours. Paris: Champion.

Tomasello, M. 2003. Constructing a Language. An Usage-based Approach to Language Acquisition. Harvard: Harvard University Press.

Torterat, F. 2009. La dictée à l'adulte, telle que pratiquée à l'école: une approche combinée des faits grammaticaux et des phénomènes prosodiques. Travaux linguistiques $d u$ Cerlico 22: 293-308.

Torterat, F. 2oroa. L'analyse multivariée des productions verbales de jeunes enfants: un élément de plus en faveur des corpus longitudinaux. Textes et Corpus 3. Accessible en ligne sur http://web.univ-ubs.fr/corpus/jlc6.html.

TORTERAt, F. 2oiob. Le recours aux corpus discursifs: difficultés et possibilités pratiques. In M. St. Rădulescu, B. Darbord et A. Solcan (eds), La méthodologie pour un apprentissage de la recherche. Bucarest: Editura Didactica si Pedagogica: 7I-90.

Torterat, F. 2oioc. La Grammaire de texte: un domaine confronté aux allants-de-soi. Le Langage et l'Homme XXXXV (2): 53-60.

Veneziano, E., Berthoud, I. et Sinclair, H. i99o. From One Word to Two Words: Repetition Patterns on the Way to Structured Speech. Journal of Child Language 17: 633-650.

Veneziano, E. et Hudelot, C. 2007. Processus discursifs dans le développement de la cohérence narrative. Actualités Psychologiques 19: 41-46. 